\title{
Exploring the Relationship between Computational Intelligence and Financial Engineering
}

\author{
Jingwei Zhong, Aijun Zuo
}

Nanchang Institute of Technology, Nanchang, Jiangxi, 330108

Keywords: computational intelligence; financial engineering; relationship

\begin{abstract}
The continuous development of modern society is no longer a society in which economic development in the past has been a trend, but is now a high-tech society with a network economy and a knowledge economy as the main growth goals. Under such a social environment background, various advanced technologies are widely used in all areas of social development needs, such as information management optimization technology, big data co-ordination, network communication technology, and all other advanced high-tech technologies. The application of computational intelligence to financial engineering is a significant step forward. It is of great significance to the improvement of the level of socio-economic development, the guarantee of people's living standards and the advancement of human society.
\end{abstract}

\section{Introduction}

Computational intelligence is a branch of modern artificial intelligence that allows machines or information systems to learn and reason automatically. Computational intelligence is a product of the integration of many different disciplines such as information science, cognitive science, and life science. It is mainly derived from the ideas of bionics and pseudo-objects. It is based on human understanding of certain natural sciences and the intelligent mechanisms of living organisms and uses computations. Numerical methods to imitate and embody human intelligence, biological intelligence, other social and natural laws. Computational intelligence recognizes and models intelligence in terms of biological evolution. According to this view, intelligence is the inheritance and variation of living things. The natural selection of development and its external environment sprouted. In the process of using the waste to back out and win the best and eliminating the fittest, the smart (mind) structure has been left behind, and the level of intellectual development has also increased. Therefore, computing intelligence is based on the intelligence of structural evolution.

In fact, computational intelligence is a model and smart tool that can be used for calculation. In an unstable environment, it is easy to directly input raw data, and then process the data through an accurate parallel expression method. Finally, a credible and highly tolerant result is quickly generated. The typical methods of computational intelligence include: 1. Fuzzy set theory, 2. Psychic networks, 3. Evolutionary calculation methods (removal algorithm, genetic regulation method, particle mass transfer method), 4. Trust network, 5 Artificial life.

The theory of computational intelligence mainly uses the following aspects: intelligent modeling, intelligent control, intelligent optimization, intelligent management, intelligent simulation, intelligent design, and manufacturing. For example, intelligent modeling, abandoning the original physical model modeling methods and mathematical models, and using the system's measured input data, operating experience, and known knowledge to assist in computational intelligence, greatly solve the complexity of modeling problems and make the model more complex. Natural and accurate. Computational intelligence is a new topic in the development of human artificial intelligence. It is one of the newest technologies in the world and is one of the cores for completing systematic, standardized, accurate, and automated equipment in all walks of life. In any case, the most advanced technological expression of smart technology is to fit the human brain both structurally and functionally. The artificial intelligence theory and technology in the future is precisely reflected in the human brain. Advanced intelligent artificial and comprehensive intelligent science and technology are the goals we strive for. Establishing the future of high intelligence and 
quality planet is our long-term ultimate goal!

\section{Overview of Financial Engineering}

Financial engineering originated in the middle and late 1980s as a new discipline in Western developed countries. In 1998, Finetti, the professor of finance at the United States, first proposed the design, development, and implementation of financial instruments and financial processes to provide realistic solutions to financial problems that have emerged or have not yet emerged. It mainly includes all kinds of financial instrument by-products and various excellent native financial instruments.

In general, the basic meaning of financial engineering is narrow and broad. Narrowly defined financial engineering mainly refers to the use of existing communication tools and superb mathematical calculations to perform various types of portfolio decomposition on various types of existing foundation fund financial products, all designed to meet the requirements of the customers. works. In the broad sense, financial engineering refers to the technical implementation means that all use engineering techniques to solve financial problems. More than financial product design also includes financial risk management, trading strategy design, pricing of financial products, and so on. This is a cross-cutting comprehensive discipline that integrates finance, engineering, statistics, and computer technology.

The top priority of financial engineering is nothing more than the application of the theory of no-arbitrage equilibrium. Due to the limitations of financial products and trading rules, it is often difficult to accurately and accurately describe changes in the supply line of assets and changes in equilibrium. However, if this asset is combined with other assets in the market, a new market equilibrium cannot be created. With a combination of risk-free returns, these current asset balances can be effectively resolved. In addition, many theories such as the Black-Scholes option pricing theory and the arbitrage pricing theory draw on the principle of no-arbitrage equilibrium pricing. From this we can see that the principle of no-arbitrage equalization is the core technology of the center of financial activities and plays a decisive role for every financial engineer.

\section{The Application of Computing Intelligence in Financial Engineering}

Financial engineering is the introduction of engineering thinking into the financial field, the comprehensive use of various methods to design, develop and implement new types of financial products, and creatively solve various financial problems. Computational intelligence technology is one of the more complete technological means of development at this stage. It has made impressive achievements in all aspects. If the technology is applied to the research of financial engineering, it will promote the development of financial engineering as a connecting bridge.

In general, the trend of stock prices is very uncertain, but in the long run, there is a certain degree of regularity. Artificial neural network can be regarded as a "black box" with self-learning function and memory function. It has considerable advantages in the face of the problem that it is impossible to establish a model study. If you use the artificial neural network toolbox, analyze and model the forecast price trend of stocks and then more accurately predict the trend of recent stocks. This is a major emerging technology in modern computing intelligence.

In modern trading decision-making, computational intelligence can fully utilize the prediction results of previous big data. Some people propose a combination of Bollinger Bands and an improved immune genetic algorithm to optimize intelligent trading decisions based on forecasting trends and actual trends. Problems that may arise in decision-making and propose solutions to specific problems. Intelligent simulation of realistic results gives you the best and the best results.

In portfolio investment, for the deficiency of Markowite's mean-variance model, computational intelligence proposes a securities portfolio investment method based on improved immune genetic algorithm. It perfects the specific solution to the mean-variance model. The return rate must conform to the normal distribution. The limitations, and through the separate analysis of the risk loss, investment income and risk compensation, to clarify the relationship between the three, but 
also by the introduction of the weighting method of preference coefficients put the relationship between the three in a balanced point. .

\section{The Problems in the Application of Computing Intelligence in Financial Engineering}

For the first time, the new concept of "financial engineering" was introduced in 1988. It has not been developed for 30 years so far. So, for now, it is not perfect. As for China's financial engineering, it is still in its infancy. It is because of unstable market factors and other obstacles in China that the development of financial engineering is seriously lagging behind. All-round integration of financial services and capital flows cannot be carried out normally. There is no way to accomplish the purpose of risk. Therefore, it seems that the development of China's financial engineering has a long way to go.

The scope of application in financial engineering has yet to be expanded and the direction of action in financial engineering needs to be improved.

The application of financial engineering has not been closely linked with international related trends.

\section{Application of Improved Intelligence in Financial Engineering}

In response to the improvement in financial engineering, we must first develop a reasonable financial policy, and only in this way can we have certain standards of behavior and ethics. Secondly, it is necessary to cultivate large-scale market consumption subjects, break the traditional theoretical constraints, and constantly discover new technologies in the course of continuous practice. We must learn to "teach students in accordance with their aptitude" and give different treatment methods to different subjects to avoid risks and maximize their benefits. . In the end, it is necessary to recruit talents from all over the world to cultivate professional talents in the field of financial engineering, to learn the essence of knowledge and understanding, to apply knowledge to real life, and to accumulate effective experience so as to promote the efficient development of the financial industry.

If an entity with an exposure risk wants to eliminate the risk, it is called the hedging pass and the original hedging relationship. A high degree of engagement with the most primitive risks must lead to a perfect naughty insurance value tool, which in theory can completely avoid risks. However, we must know that perfection is impossible. Generally speaking, a well-designed hedge insurance can play a certain role in improving, but it is only a different role, but we must also realize that there are some sets of designs that are very unreasonable. The period of insurance hedges will only lead to higher risks.

Speculation can be used to forecast through the sensitivity and trends of future market changes. Future market trends, an investment in uncertainties, may at the same time generate a new risk exposure. Generally, there are two kinds: "You want to suppress first," and "You want to suppress first." If derivative tools such as computational intelligence are used during speculation, financial speculation can be of great help.

The price of a commodity follows a certain value rule, that is, value determines the price rule, the price fluctuates along with the value, and is also affected by changes in the supply and demand of the market and other factors. Based on these variables, the price also changes, so the same The product also presents different prices. Do not be afraid that usually these price deviation gaps are relatively small, giving arbitrage some room. When there is a price deviation, the computing intelligence will automatically buy at a low cost and sell it at a high price in order to earn an intermediate price difference.

Financial engineering can propose different technical references and solutions for different trading entities and exposure risks, and rearrange and combine financial instruments. If we say that a company's income and expenditure are not the same as the type of currency, it is possible to match the transaction proportions through exchange of currencies and avoid large amounts of economic losses. With the rapid development of economy, computing intelligence is increasingly used in the 
financial industry. In addition, the status of computational intelligence in financial engineering is increasingly unshakeable. It can not only increase efficiency, but also enable financial macroeconomic regulation and control to promote the future development of China's economy. This is a tool for financial engineering.

\section{Conclusion}

After the introduction of computational intelligence in financial engineering, various methods were used to design, develop, and implement emerging financial engineering products. The feasibility of solving various financial problems was calculated. Computational intelligence technology is a relatively modern technological means. one. The achievement of gratifying achievements in financial engineering has produced a great promotion effect through the introduction of this technology for the economic development of financial engineering. For now, computational intelligence is still lacking in financial engineering. In the future, we need to do more and the revolution is not yet successful. Comrades still need to work hard.

\section{References}

[1] Yan Xiaogang. Nerve Computing Science[J]. 2015(01)

[2] Jiang Zejun. Intelligent Computing - Theory of Rough Set Theory, Fuzzy Logic, Neural Network and Its Application [J]. 2015(02)

[3] Pan Zhengjun, Kang Lishan. Evolutionary calculation[D].2015(05) 